NASZA DERMATOLOGIA Online OUR DERMATOLOGY Online

Georgia Dermatopathology

Associates, Atlanta, Georgia, USA

(MSH, AMAV); Mineros SA, Medellin Colombia SA; Embassy of Japan in Colombia, University of Antioquia.

Competing Interests: None

\section{CYCLO-OXYGENASE 2 IS PRESENT IN THE MAJORITY OF LESIONAL SKIN FROM PATIENTS WITH AUTOINMUNE BLISTERING DISEASES}

\author{
Ana Maria Abreu Velez ${ }^{1}$, Juliana Calle Isaza ${ }^{2}$, Michael S. Howard ${ }^{1}$ \\ ${ }^{1}$ Georgia Dermatopathology Associates, Atlanta, Georgia, USA \\ ${ }^{2}$ Medellin General Hospital, Medellin, Antioquia, Colombia, S.A. \\ Corresponding author: Ana Maria Abreu Velez, MD PhD \\ abreuvelez@yahoo.com
}

\begin{abstract}
Introduction: The in situ immune response within skin biopsies from patients affected by autoimmune skin blistering diseases (ABDs) is not well characterized.

Aim: Based on the fact that the ABD immune response is considered an adaptive immune response, both an innate immune response and inflammation would be expected in these diseases. Our investigation investigates the presence of cyclo-oxygenase-2 (COX-2), since this enzyme is commonly involved in innate immune responses.

Methods: We utilized immunohistochemistry (IHC) to evaluate the presence of COX-2 in lesional skin biopsies of patients affected by ABDs. We tested 30 patients with endemic pemphigus foliaceus (EPF), 15 controls from the endemic area, and 15 biopsies from healthy controls from the USA. We also tested archival biopsies from patients with selected ABDs, including 20 patients with bullous pemphigoid, 20 with pemphigus vulgaris, 8 with pemphigus foliaceus and 12 with dermatitis herpetiformis.

Results: Most ABD biopsies stained positive for COX-2 in the lesional blister and/or the dermal inflammatory infiltrate, accentuated in the upper neurovascular plexus. In BP and EPF, the COX-2 staining was also seen in the sweat glands. All controls were negative.

Conclusions: We document that COX-2 is expressed in lesional skin of patients with ABDs.
\end{abstract}

Key words: Cyclo-oxygenase 2; autoimmune skin diseases; endemic pemphigus foliaceus

Abbreviations and acronyms: Bullous pemphigoid (BP), immunohistochemistry (IHC), direct and indirect immunofluorescence (DIF and IIF), hematoxylin and eosin (H\&E), basement membrane zone (BMZ), intercellular staining between keratinocytes (ICS), pemphigus vulgaris (PV), cicatricial pemphigoid (CP), autoimmune blistering skin diseases (ABDs), fogo selvagem (FS), endemic pemphigus foliaceus in El-Bagre, Colombia (El Bagre-EPF).

Ana Maria Abreu Velez, Juliana Calle Isaza, Michael S. Howard: Cyclo-oxygenase 2 is present in the majority of lesional skin from patients with autoinmune blistering diseases. Our Dermatol Online. 2013; 4(4):476-478.

\section{Highlights:}

\section{What is known:}

B lineage lymphocytes and complement play an important role in ABDs, by producing antibodies that are deposited in lesional skin and possibly leading to blister formation.

\section{Introduction}

Multiple therapies have been utilized for the treatment of cutaneous autoimmune blistering skin diseases (ABDs). Steroids represent a commonly utilized therapy, because many autoimmune disorders are B-lymphocyte mediated processes depositing autoantibodies and complement deposits in the skin. Well documented correlations exist between titers of autoantibodies and the clinical severity of the diseases [1-3].

\section{What does the current study add?}

Immune system markers such cyclo-oxygenase- 2 seem to be expressed in the majority of lesional skin of patients with ABDs, and may represent a consistent feature of the inflammation commonly present in these diseases.

The present investigation aims to study an inducible marker of the innate immune response, specifically the presence of cyclo-oxygenase 2 (COX-2); this enzyme generates inflammatory prostanoids, and inflammation is a hallmark of multiple ABDs. Thus, we studied the in situ immune response by performing immunohistochemistry (IHC) stains on lesional skin biopsies for COX-2. 


\section{Materials and Methods}

\section{Subjects of study:}

We tested 30 biopsies from patients affected by EPF in El Bagre, Colombia, South America (E1 Bagre-EPF) and 15 normal controls from the endemic area [4-8]. We also utilized 15 control skin biopsies from cosmetic reduction plastic surgery patients in the USA, taken from the chest and/or abdomen. Biopsies were fixed in $10 \%$ buffered formalin, then embedded in paraffin and cut at 4 micron thicknesses. The tissue was then submitted for hematoxylin and eosin (H\&E) and IHC staining. In addition, we also tested biopsies from the archival files of two private laboratories led by board certified dermatopathologists in the USA; these patients underwent initial diagnostic biopsies, and therefore were likely not taking immunosuppressive medications at the time of biopsy. We evaluated 20 biopsies from bullous pemphigoid (BP) patients, 20 from patients with pemphigus vulgaris (PV), 8 patient biopsies with pemphigus foliaceus (PF) and 12 from patients with dermatitis herpetiformis $(\mathrm{DH})$. For all of the El Bagre area patients and controls, we obtained written consents as well as Institutional Review Board permission from the local hospital. The archival biopsies were IRB exempt due to the lack of patient identifiers. In both dermatopathology laboratories, each biopsy also was sent for direct immunofluorescence as previous described [3], for correlation with the $\mathrm{H} \& \mathrm{E}$ diagnoses.

\section{Quantitative digital morphometry and IHC staining:}

The staining intensity of the antibodies was also evaluated in a semiquantitative mode by an automated computer image analysis system, designed to quantify IHC staining in hematoxylincounterstained histologic sections. Slides were scanned with a ScanScope CS system, utilizing brightfield imaging. IHC staining was performed as previously described. For IHC, we utilized a Dako monoclonal mouse anti-human COX-2 antibody, clone CX-294; staining was performed as previously described [4-7].

\section{Statistical analysis:}

For statistical analysis, the non-parametric Mann-Whitney U-test was used to calculate significant levels for all measurements. Values of $p<0.05$ were considered statistically significant.

\section{Result}

We noted that 26/30 patients with EPF were positive in the epidermis in spot areas of the corneal layers, around the neurovascular areas of eccrine and hair follicles. Only 2 controls from the endemic area showed some corneal reactivity $(p<0.05)$. Further, 17/20 biopsies from BP patients were positive for COX2 in the sweat glands, under the blisters and along the bases of the blisters. Some reactivity was seen in the corneal layers $(p<0.05)$. Reactivity was seen in the upper neurovascular plexus of the dermis, and in some type of junction between endothelial cells and the extracellular dermal matrix (Fig. 1). In patients with PV, $16 / 20$ biopsies were positive in the upper dermal inflammatory infiltrate, and around the epidermal blisters $(p<0.05)$. In patient biopsies with $\mathrm{PF}, 5 / 8$ were positive in the epidermis in spotty areas of the corneal layers, and around the neurovascular supplies of eccrine glands and hair follicles. In 9/12 patients with $\mathrm{DH}$, positive staining was noted, mostly under the BMZ $(p<0.05)$. In Figure 1, we highlight the most common patterns of positivity found in these patients.

\section{Discussion}

Because adaptive immunity has been demonstrated to be play a pathogenic role in ABDs, it is important to note that innate immunity is the first step in an adaptive immune response. Unfortunately, few studies have specifically studied molecules involved in the adaptive immune response in ABDs. Hallmark pathologic events in ABDs include vasodilation of the microcirculation, resulting in increased blood flow to the affected area. The vasodilation is responsible for the heat and redness that occurs at sites of inflammation. In ABDs, we also often see increases in the permeability of upper dermal blood vessels, promoting the movement of fluid and plasma proteins into the interstitial areas [8]. In ABDs, chemotatic neutrophils, monocytes and other white blood cells manifest as an inflammatory infiltrate. Activated complement, another innate immune marker found in pemphigus blister fluids, suggests a pathogenetic role for complement in this disorder [9].

In our study, we investigate the immune response induction marker COX-2, a key enzyme of arachidonic acid metabolism. Cyclooxygenase exists as two distinct isoforms. COX-1 is constitutively expressed in most tissues, whereas COX-2 is inducibly expressed at sites of inflammation. An additional recently documented isoform, $\mathrm{COX}-3$, is produced via alternative splicing of COX-1 and has also been described [10]. COX-2 is also inducibly expressed in neoplastic tissues. Prostanoids are produced by many cell types, and act on target cells through specific $G$ protein-coupled receptors. Although prostanoids have traditionally been considered acute inflammatory mediators, studies using knockout mice show that prostanoids may regulate selected aspects of both innate and adaptive immunity; such a dual role may be the case in ABDs. Each prostanoid, depending on which receptor it acts on, exercises specific effects on immune system cells such as macrophages, dendritic cells, and $\mathrm{B}$ and $\mathrm{T}$ lymphocytes, often in concert to microbial ligands and cytokines. These cellular actions affect the strength, quality, and duration of immune responses. Prostanoids play a critical role in immunopathology, via inflammation, autoimmunity and cancer pathophysiology [8].

We found limited specific information regarding autoimmune diseases and the expression of COX-2. In oral lichen planus, increased levels of COX-2 have been reported [11]. In experimental autoimmune encephalomyelitis, celecoxib (a new generation COX-2 inhibitor) retards inflammation [12]. Since our study utilized archival biopsies, the requisition forms did not contain specific information regarding administration of immunosuppressive agents. The precise role of this enzyme in $\mathrm{ABDs}$ requires further investigation since this was a pilot study. We thus recommend larger studies, utilizing only patients known to have no immunosuppressive therapy to study the role of this molecule in ABDs.

We found no specific studies regarding the presence of COX2 in ABDs. For this reason and because our study has a small sample size, we recommend larger studies to further define the role of COX-2 in these disorders.

\section{Conclussion}

We suggest that molecules such as complement and COX-2 are present in the majority of skin lesional biopsies from patients with ABDs; further, it is possible that COX-2 may consistently contribute to the inflammation seen in these diseases. Recent data has also demonstrated that prostanoids regulate selected aspects of both innate and adaptive immunity, and this may also be the case in ABDs. 

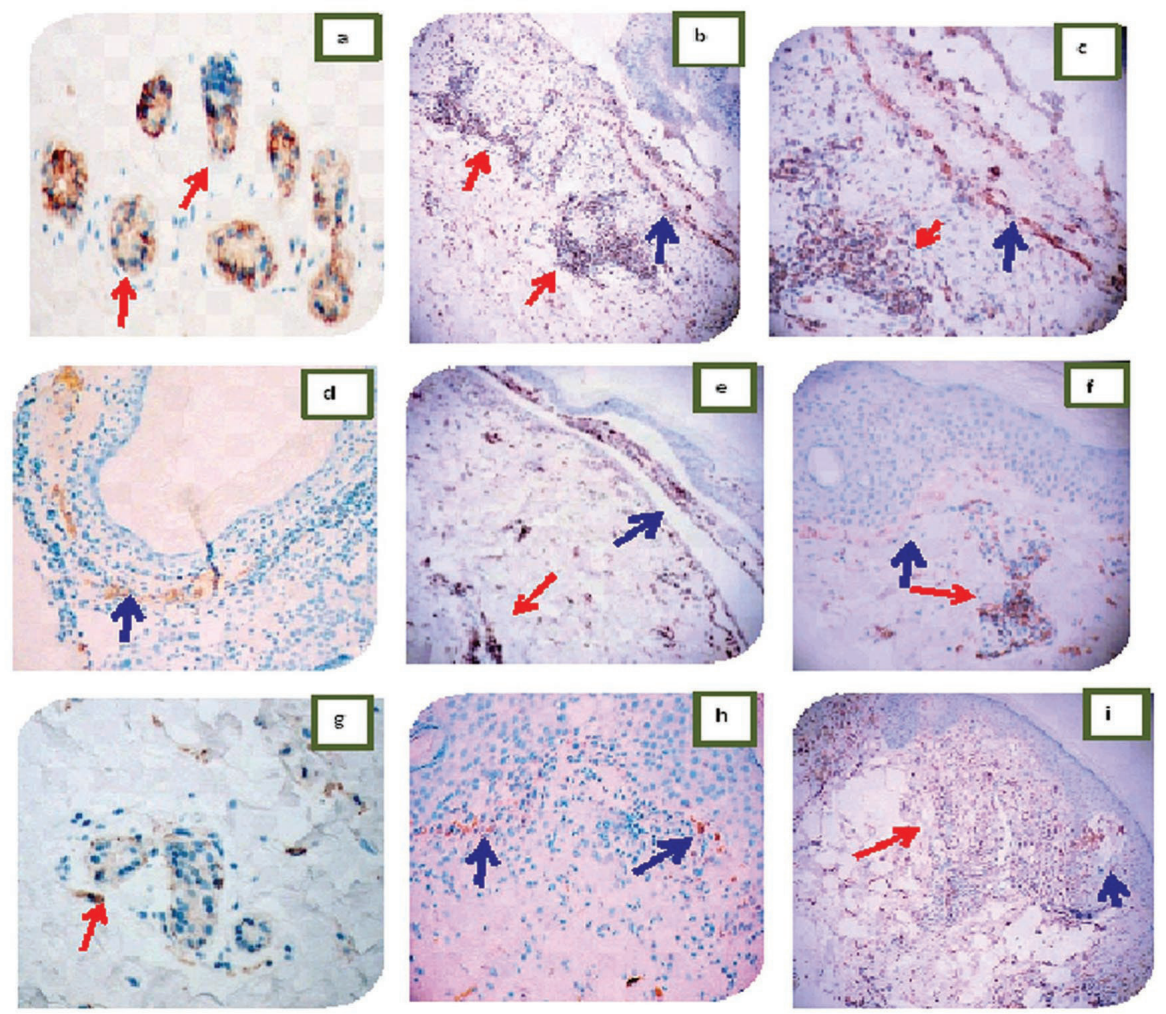

Figure 1. a. COX-2 positive IHC staining in the eccrine sweat glands of a BP patient (brown staining; red arrows). $\mathbf{b}$ and c. Positive COX-2 IHC staining in a linear manner in the base of the blister in a BP patient (brown staining; dark blue arrows), as well as along the upper dermal neurovascular plexus (brown staining; red arrows; 200X and 400X, respectively). d. A representative PV case, showing positive IHC staining with COX-2 at the separation plane of a suprabasilar blister (brown staining, dark blue arrow). e. COX-2 positive IHC staining (brown staining) in a second case of PV. The red arrow shows positivity at the upper dermal inflammatory infiltrate, and the dark blue arrow within the blister. f. A DH case with positive COX-2 IHC staining (brown staining); some weak linear staining is noted under the basement membrane zone (BMZ) (red arrow) but also around upper dermal blood vessels (blue arrow). g. COX-2 positive IHC staining within eccrine sweat gland ducts of a PV patient (brown staining; red arrow). h. Positive linear IHC staining with COX-2 in a DH biopsy (brown staining; dark blue arrows). i. COX-2 positive IHC staining inside the epidermal blister in a PV case (brown staining, dark blue arrow), and also strong positivity in the upper dermal inflammatory infiltrate (red arrow).

\section{Acknowledgements}

To assisting personnel at the Hospital Nuestra Senora del Carmen in El Bagre, to the personal in Mineros SA, the major of El Bagre, the Secretary of the Health in El Bagre and the community; and to Jonathan S. Jones HT (ASCP) at Georgia Dermatopathology Associates for excellent technical assistance.

\section{REFERENCES}

1. Bystryn JC, Habib N: Treatment options in pemphigus. G Ital Dermatol Venereol. 2009;144:351-61.

2. Rye B, Webb JM: Autoimmune bullous diseases. Am Fam Physician. 1997;55:2709-18.

3. Beutner EH: The development of immunofluorescence and the immunopathology of the skin. Int J Dermatol. 2003;42:99-109.

4. Abrèu-Velez AM, Beutner EH, Montoya F, Bollag WB, Hashimoto $\mathrm{T}$ : Analyses of autoantigens in a new form of endemic pemphigus foliaceus in Colombia. J Am Acad Dermatol. 2003;49:609-14.

5. Abreu-Velez AM, Robles EV, Howard MS: A new variant of endemic pemphigus foliaceus in El-Bagre, Colombia: the HardyWeinberg-Castle law and linked short tandem repeats. N Am J Med Sci. 2009;1:169-78.

6. Abrèu-Velez AM, Hashimoto T, Bollag WB, Tobón Arroyave S,
Abrèu-Velez CE, Londoño ML, et al: A unique form of endemic pemphigus in northern Colombia. J Am Acad Dermatol. 2003;49:599608.

7. Abreu-Velez AM, Howard MS, Yi H, Gao W, Hashimoto T, Grossniklaus HE: Neural system antigens are recognized by autoantibodies from patients affected by a new variant of endemic pemphigus foliaceus in Colombia. J Clin Immunol. 2011;31:356-68. 8. Hirata T, Narumiya S: Prostanoids as regulators of innate and adaptive immunity. Adv Immunol. 2012;116:143-74.

9. Jordan RE, Day NK, Luckasen JR, Good RA: Clin Exp Immunol. Complement activation in pemphigus vulgaris blister fluid. 1973; 15:53-63.

10. Vane JR, Mitchell JA, Appleton I, Tomlinson A, Bishop-Bailey $\mathrm{D}$, Croxtall $\mathrm{J}$, et al: Inducible isoforms of cyclooxygenase and nitric oxide synthase in inflammation. Proc Natl Acad Sci USA. 1994;91:2046-50.

11. Danielsson K, Ebrahimi M, Wahlin YB, Nylander K, Boldrup $\mathrm{L}$ : Increased levels of COX-2 in oral lichen planus supports an autoimmune cause of the disease. J Eur Acad Dermatol Venereol. 2012;26:1415-19.

12. Miyamoto K, Miyake S, Mizuno M, Oka N, Kusunoki S, Yamamura T: Selective COX-2 inhibitor celecoxib prevents experimental autoimmune encephalomyelitis through COX-2independent pathway. Brain. 2006;129:1984-92.

Copyright by Ana Maria Abreu Velez, et al. This is an open access article distributed under the terms of the Creative Commons Attribution License, which permits unrestricted use, distribution, and reproduction in any medium, provided the original author and source are credited. 